\title{
Sintomas depressivos, de ansiedade e de estresse em habitantes de munícipio realocado por barragem
}

\author{
Franciele Luísa Pozzebon \\ Vinícius Renato Thomé Ferreira \\ Faculdade Meridional, RS, Brasil
}

\begin{abstract}
Resumo
O aumento da necessidade de energia elétrica faz com que a instalação de usinas hidrelétricas seja um investimento importante; entretanto, os impactos ambientais e humanos decorrentes da instalação de barragens precisam ser apropriadamente avaliados. Buscou-se investigar a prevalência de sintomas depressivos, de ansiedade e de estresse de uma população que passou pelo processo de realocação devido à construção de uma barragem para usina hidrelétrica $(n=50)$ em comparação com um município onde não houve este processo $(n=50)$. Foi identificado que, vinte anos após a instalação da barragem e realocação do município, os escores de sintomas depressivos, de ansiedade e de estresse estão na faixa da população não clínica, e foram mais elevados no município de comparação do que no município onde a barragem foi instalada. Estudos sobre a instalação de barragens e realocação humana precisam considerar, além dos impactos ambientais, seus reflexos sobre a saúde mental das pessoas.
\end{abstract}

Palavras-chave: Barragem; Sintomas depressivos; Sintomas de ansiedade; Sintomas de estresse; Avaliação psicológica.

\section{Depressive symptoms, anxiety and stress in population in municipality reallocated by dam}

\section{Abstract}

The increased need for electricity, especially with less environmental impact, makes the installation of hydroelectric plants an essential alternative. However, environmental and human impacts of the dam installation need to be evaluated in depth. We had investigated the prevalence of depressive, anxiety and stress symptoms of a population that has passed by the relocation process due to construction of a dam for hydroelectric power plant $(n=50)$ compared to another county which had not been passed by this process $(n=50)$. It was identified that scores of depressive symptoms, anxiety and stress are in the range of non-clinical population twenty years after the relocation of the city, and were higher in the county where the dam was not installed. Studies on the impact of the installation of dams and human allocation need to consider the assessment of depressive symptoms, anxiety and stress to identify possible impacts on the mental health.

Keywords: Dam; Depressive symptoms; Anxiety symptoms; Stress; Psychological assessment.

\section{Síntomas depresivos, ansiedad y el estrés de la población en el municipio de reubicado por presa}

\section{Resumen}

La mayor necesidad de energía eléctrica hace que la instalación de centrales hidroeléctricas es una inversión importante; Sin embargo, los impactos ambientales y humanos derivados de la instalación de presas deben ser evaluados correctamente. Hemos tratado de investigar la prevalencia de síntomas depresivos, ansiedad y el estrés de una población que pasa el proceso de reubicación debido a la construcción de una presa para hidroeléctricas $(n=50)$ en comparación con un municipio donde no había este processo $(\mathrm{n}=50)$. Se identificó que veinte años después de la instalación de la presa y la reubicación de la ciudad, las puntuaciones de los síntomas de depresión, la ansiedad y el estrés están en el rango de la población no clínica, y fueron mayores en el municipio de la comparación. Los estudios sobre la instalación de presas y reubicación humana deben tener en cuenta, además de los impactos ambientales, sus efectos sobre la salud mental.

Palabras clave: Presa; Síntomas depresivos; Síntomas de ansiedad; Síntomas de estrés; Evaluación psicológica. 


\section{Introdução}

Com a necessidade de aumento da matriz energética no Brasil, é necessária a construção de novas usinas para minorar os riscos de racionamento (Passos \& Praxedes, 2013). A geração de energia através de hidrelétricas consiste na produção de eletricidade por meio do aproveitamento do potencial hidráulico obtida com a construção de usinas em rios que possuam grande vazão de água e que tenham desníveis em seu curso. A eletricidade produzida por hidrelétricas é considerada uma fonte de energia limpa, porém não está isenta de impactos ambientais e sociais, uma vez que a formação do lago artificial inunda vastas áreas gerando por vezes problemas no meio ambiente físico e a eventual necessidade de realocação das pessoas (Francisco, 2011).

Os empreendimentos hidrelétricos implantamse dentro do interesse coletivo de uma população por ascender, em oferta de energia, a qualidade de vida e a capacidade de produção. Contudo, além da necessidade de se considerar os benefícios, devem ser analisados os impactos da construção de hidrelétricas (Souza, 2000). O principal impacto analisado costuma ser o do ambiente físico, pela produção de alagamento de importantes áreas e o desaparecimento do habitat de animais (Borges \& Silva, 2011). A modificação de um ambiente lótico (rios) em ambiente lêntico (água parada das barragens) gera prejuízos na ictiofauna (espécies de peixes da região), alterando o equilíbrio ambiental (Mendonça, 2011). Com o propósito de minimizar os efeitos prejudiciais, no Brasil o procedimento de avaliação de impactos está ligado ao processo do licenciamento ambiental, que permite avaliar a viabilidade ambiental do empreendimento com a utilização de medidas mitigadoras para os prejuízos identificados para tornar a construção viável (Costa, Locks, \& Matos, 2010).

Além dos impactos ambientais, as construções costumam gerar impacto humano. Se considerarmos que o comportamento é influenciado pelo ambiente da mesma forma como ele o influencia (Skinner, 2003), deve-se concluir que as mudanças no ambiente possuem um potencial para causar melhoras ou sofrimentos na vida das pessoas dependendo de como essas mudanças são gerenciadas. O ambiente ecológico afeta diretamente a pessoa em desenvolvimento assim como as relações que esta possui com o sistema (Bronfenbrenner, 1996), sendo portanto imprescindível relacionar o ambiente e o ser humano no contexto da realocação pelas hidrelétricas. Um lugar possui valores, representações e um significado particular para cada sujeito, apresentando substancial carga afetiva (Chaves,
2000; Borges \& Silva, 2011), e alterações ambientais interferem significativamente na saúde mental.

Há muito o que se aprimorar na avaliação dos impactos humanos na saúde mental pela instalação de barragens, visto que atualmente a quase totalidade dos estudos priorizam os impactos ambientais (Rossouw, 2008). Não avaliar o impacto sobre a saúde mental das populações atingidas fará o estado e a iniciativa privada ignorar importantes investimentos futuros em saúde e qualidade de vida após a instalação, gerando a necessidade de mais investimento para mitigar estes quadros. Entre os impactos humanos ocasionados pela realocação devido à instalação de barragens, podem ocorrer alterações na qualidade de vida e o aparecimento de sintomas depressivos e de ansiedade, tendo em vista as enormes modificações geradas na comunidade e nas pessoas individualmente. Estudos dos impactos da realocação sobre sintomas depressivos e de ansiedade devido à instalação de barragens são escassos, e mais raros ainda os estudos que acompanham esta população ao longo do tempo; contudo, já foi possível identificar que mesmo após 50 anos do processo de realocação devido à instalação de barragens podem ser identificados efeitos na comunidade, como por exemplo maiores referências de memória ao passado (Pirta, Chandel, \& Pirta, 2014; Kedia \& Van Willigen, 2001).

O município de Itá, no estado de Santa Catarina, passou há cerca de 20 anos atrás pelo processo de realocação da população devido à instalação de uma barragem, afetando a população urbana e a rural. Itá está localizada no rio Uruguai, na divisa dos estados de Santa Catarina (município de Itá) e Rio Grande do Sul (município de Aratiba), na região sul do país (Hermes, 2013). A Usina Hidrelétrica de Itá (UHE) foi o primeiro empreendimento hidroelétrico no trecho brasileiro do rio Uruguai e um dos maiores da região sul do Brasil. Com a construção da UHE, 11 municípios foram atingidos, sendo sete em Santa Catarina (Itá, Piratuba, Arabutã, Concórdia, Alto bela Vista, Ipira, Peritiba) e quatro no Rio Grande do Sul (Aratiba, Mariano Moro, Severiano de Almeida e Marcelino Ramos). Itá foi o município mais atingido, sendo necessário relocar a cidade antiga para uma área distante seis quilômetros das margens do rio, visto que as águas do reservatório inundaram completamente o perímetro urbano (Viana, 2003). Esse processo de realocação da população, na época, gerou certo impacto, tendo em vista que trouxe consigo uma importante mudança física na vida das pessoas e, por consequência, modificação de hábitos, costumes, tradições e na memória coletiva por apagar referências físicas (Elementos Culturais da Área do Reservatório, 2003). Consequentemente, houve uma luta para manter a identidade da comunidade e se 
estruturar com características próprias dentro da nova realidade.

É fundamental identificar o impacto de grandes mudanças ambientais sobre o comportamento humano, especialmente no que diz respeito a sintomas depressivos, de ansiedade e sobre o estresse, visto que estes fatores estão associados diretamente à maioria, senão a todos, os transtornos mentais (Sadock, Sadock \& Ruiz, 2016). Os sintomas depressivos referem-se a prejuízo nas emoções, especialmente pela presença de tristeza (humor deprimido) e perda do interesse e prazer em atividades do dia a dia, e incluem: alterações significativas de peso em período curto de tempo, alterações do sono (insônia e/ou hipersonia), agitação ou retardo psicomotor, fadiga, sentimentos de culpa e inutilidade, redução da capacidade de pensar e podem ocorrer pensamentos recorrentes de morte (American Psychiatric Association, 2014). Os sintomas de ansiedade referem-se a sensações corporais difusas e desagradáveis acompanhadas de um sentimento de apreensão, incluindo aumento da frequência cardíaca, desconforto estomacal e agitação psicomotora (Sadock, Sadock, \& Ruiz, 2017) que ocorrem por antecipação a algum evento ameaçador. O estresse, por sua vez, diz respeito à exposição de eventos que excedem a capacidade de controle ou manejo do organismo, gerando sintomas comportamentais importantes, como por exemplo a presença crônica de ansiedade, medo, comportamentos de evitação e pesadelos repetitivos (American Psychiatric Association, 2014; Sadock, Sadock, \& Ruiz, 2017). A identificação da prevalência destes sintomas auxilia o estado no planejamento de intervenções de prevenção e tratamento (Lancetti \& Amarante, 2006).

Tendo em vista a baixa frequência de estudos sobre o impacto da construção das barragens e sua relação com a saúde mental, e considerando-se o impacto humano gerado pelo processo de instalação de barragem e realocação de populações, objetivou-se avaliar se a prevalência de sintomas depressivos, tríade cognitiva, sintomas de ansiedade e de estresse de uma amostra da população de Itá 20 anos após a instalação da barragem e consequente realocação populacional, difere em comparação com uma amostra de outro município que não passou pelo processo de realocação (Passo Fundo). Com isso, pretendeu-se identificar se haveriam diferenças estatisticamente significativas (em nível de $\mathrm{p} \leq 0,05$ ) entre a prevalência destes sintomas nos dois municípios.

\section{Método}

Participaram do estudo 50 pessoas do município de Itá, tendo ou não passado pelo processo de realocação devido à construção da barragem, com idade igual ou superior a 18 anos e que residam na cidade, sendo este o principal critério de inclusão no estudo. A título de comparação, o desempenho dos participantes desta amostra foi analisado em relação a 50 pessoas residentes num município que não passou pela experiência de realocação por instalação de barragem, totalizando 100 participantes. Como o objetivo deste estudo visou comparar as amostras dos dois municípios, não foi o foco do mesmo investigar os sintomas de ansiedade e estresse especificamente nos munícipes que passaram pela realocação.

Foram utilizados como instrumentos de pesquisa um questionário sociodemográfico com o intuito de traçar o perfil dos participantes da pesquisa; a escala de depressão do Center for Epidemiological Studies (CES-D), escala autoaplicável composta de 20 itens que mensuram sintomas depressivos, numa escala Likert de quatro pontos (Silveira \& Jorge, 1998); o Inventário de Ansiedade Traço-Estado (IDATE), desenvolvido para identificar e distinguir ansiedade traço e ansiedade estado, com vinte afirmativas, numa escala Likert de quatro pontos (Fioravanti, Santos, Maisonette, Cruz, \& Landeira-Fernandez, 2006); a escala de estresse percebido (EEP), que avalia sintomas de estresse e é composta por 14 questões numa escala Likert de cinco pontos (Luft, Sanches, Mazo, \& Andrade, 2007); e as escalas de levantamento da intensidade de sintomas depressivos (LIS-D), de ansiedade (LIS-A) e de estresse (LIS-E), que ainda se apresentam em fase de validação. O LIS-D tem por objetivo ser um instrumento de autoavaliação para averiguar a intensidade de sintomas depressivos, composto por 35 questões que avaliam oito dimensões relacionadas diretamente com os sintomas depressivos: sintomas afetivos, de comportamento, de pensamento, sintomas somáticos e a tríade cognitiva, composta pela visão de si, visão do mundo e visão do futuro. O LIS-A é constituído de 34 questões que avaliam sintomas de ansiedade, e o LIS-E é composto por 25 questões que avaliam sintomas de estresse. Todas as escalas LIS apresentam uma pontuação Likert de 5 pontos (variando de 0 a 4), e quanto maior a pontuação, maior a presença de sintomas (Ferreira, 2013; Ferreira, 2014a; Ferreira, 2014b).

Os dados foram inicialmente tratados pela estatística descritiva, informando frequências e porcentagens dos dados demográficos dos participantes, além do desempenho nos instrumentos. Em seguida, procedeu-se à análise inferencial dos dados, com a utilização do teste de Mann-Whitney (U) e o teste de correlação de Spearman (rho, $\rho$ ), considerando-se que os instrumentos oferecem dados de natureza ordinal (não paramétrica). 
Além disso, o teste escolhido dispensa distribuição normal (De-Moivre-Laplace-Gauss) e equivalência de variância (Lopes, 1999; Lewin \& Fox, 2004; Dancey \& Reidy, 2013), sendo uma boa escolha para o tratamento dos dados.

\section{Procedimentos}

Inicialmente entrou-se em contato com a Casa da Cultura do município de Itá, e identificou as pessoas que participaram ou não do processo de relocação da cidade em função da implantação da UHE. Posterior ao levantamento desses dados foi iniciado o processo de pesquisa de campo, onde houve o convite para a participação do estudo, explicando-se o objetivo da pesquisa, o Termo de Consentimento Livre e Esclarecido e convidou-as a participar da pesquisa, de forma não sistemática. À medida em que as pessoas iam aceitando participar do estudo, os formulários foram aplicados. Também fora averiguado a disponibilidade de horários para responder aos instrumentos da pesquisa. A coleta de dados foi realizada na residência dos referidos participantes, tendo sido complementada com outros moradores do município. A amostra de Passo Fundo foi obtida de um banco de dados previamente coletado, havendo uma seleção pseudoaleatória dos dados. A pesquisa fez parte de um projeto maior, previamente aprovado por comitê de ética em pesquisa (CEP) (CAAE 07699512.9.00005319). Após a coleta dos dados pela aplicação dos instrumentos, procedeu-se a análise estatística.

\section{Resultados}

A descrição da amostra apontou para uma maior presença de mulheres respondendo à pesquisa $(\mathrm{n}=61,61 \%)$. A escolaridade predominante foi de ensino médio completo $(n=46,46 \%)$, e de pessoas predominantemente casadas ou em união estável $(\mathrm{n}=58,58 \%)$. A maioria das pessoas declarou religião cristã $(n=90,90 \%)$, e referiu estar trabalhando $(n=80$, $80 \%$ ). A atividade predominante dos respondentes de Itá foi de aposentados $(n=8,8 \%)$, enquanto que a amostra de Passo Fundo constituiu-se principalmente de profissionais da área da saúde $(\mathrm{n}=27,27 \%)$. A renda familiar mais frequente dos participantes de Itá foi entre 5 e 6 salários-mínimos (SM) $(n=17,17 \%)$ e com idade média dos respondentes de 47,33 anos (desviopadrão 17,64 anos, nível de confiança da média a 95\% de 2,69), enquanto que a amostra de Passo Fundo apresentou maior frequência de renda entre 3 e $4 \mathrm{SM}$ $(\mathrm{n}=21,21 \%)$, com idade média de 31,82 anos (desviopadrão 7,36 anos, nível de confiança da média a 95\% de 1,04) (ver Tabela 1, adiante).
Para a análise, definiu-se a priori o valor de $\mathrm{p} \leq 0,05$ como representando significância para estabelecer diferenças estatisticamente significativas nas testagens conduzidas. O desempenho da amostra de Itá e do município de comparação foram comparadas utilizandose a prova estatística de Mann-Whitney. Considerou-se o valor de $\mathrm{p}$ a partir de uma hipótese bicaudal, ou seja, que não previsse a direção da diferença entre as duas amostras.

Os escores de sintomas depressivos, de ansiedade e de estresse apresentaram escores dentro da faixa de sintomas compatíveis com a população não clínica. O CES-D apontou para ausência de sintomas clínicos de depressão (valores acima de 17 pontos sugerem sintomas depressivos; a mediana das duas amostras apontou para o valor de 11,5 pontos), tanto quanto o IDATE ( sintomas na faixa de médio-baixos, que variam de 30 a 39 pontos, ficando as amostras na faixa de 37 pontos medianos) e a EEP (sem dados normativos; 22 pontos medianos apresentados pelas amostras). As escalas LIS para sintomas depressivos, de ansiedade e de estresse ainda estão em fase de validação, não apresentando valores normativos; contudo, houve a identificação das seguintes correlações (Spearman): entre o CES-D e o LIS-D, $\rho=0,72(<0,0001)$, entre o IDATE e o LIS-A, $\rho=0,66(<0,0001)$, e entre o EEP e o LIS-E $\rho=0,63(<0,0001)$. Estes valores podem então sugerir que os valores das escalas LIS também apontam para valores compatíveis com os da população não clínica.

A comparação das duas amostras aponta para a existência de diferenças estatisticamente significativas nas amostras de Itá e da cidade de comparação para a visão de si do LIS-D, para os sintomas de ansiedade do IDATE e para os sintomas de estresse do EEP, todas com mais prejuízo para a amostra do município de comparação. Em relação à visão de si, a diferença encontrada atingiu um valor de $p=0,05$, enquanto que no IDATE e no EEP estes valores foram de $p=0,02$. Para o CES-D, não foram encontradas diferenças nos escores $(p=0,11)$, e no LIS-A e no LIS-E todos os demais escores das dimensões apontaram para a ausência de diferenças estatisticamente significativas (valores de $p>0,05$ ) (ver Tabela 2).

Praticamente todos os escores dos instrumentos favoreceram a população de Itá, com exceção dos sintomas de pensamento, visão de mundo e visão de futuro do LIS-D (com medianas empatadas, respectivamente, em 3, 4 e 3). Apenas houve mais prejuízo, embora não estatisticamente significativo através das medianas calculadas, para os escores do LIS-A $(p=0,92)$ e do LIS-E $(p=0,65)$, favorecendo ligeiramente a amostra do município de comparação. 
TABELA 1

Descrição dos dados dos participantes do estudo

\begin{tabular}{|c|c|c|c|c|c|c|c|}
\hline Variável & Item & Barragem & $\%$ & Comparação & $\%$ & Total & $\%$ \\
\hline \multirow[t]{3}{*}{ Sexo } & Feminino & 28 & 56 & 33 & 66 & 61 & 61 \\
\hline & Masculino & 22 & 44 & 17 & 34 & 39 & 39 \\
\hline & Total & 50 & 100 & 50 & 100 & 100 & 100 \\
\hline \multirow[t]{9}{*}{ Escolaridade } & Não respondeu & 2 & 4 & 0 & 0 & 2 & 2 \\
\hline & Fundamental incompleto & 8 & 16 & 5 & 10 & 13 & 13 \\
\hline & Fundamental completo & 2 & 4 & 1 & 2 & 3 & 3 \\
\hline & Médio incompleto & 2 & 4 & 0 & 0 & 2 & 2 \\
\hline & Médio completo & 19 & 38 & 27 & 54 & 46 & 46 \\
\hline & Superior incompleto & 2 & 4 & 11 & 22 & 13 & 13 \\
\hline & Superior completo & 6 & 12 & 4 & 8 & 10 & 10 \\
\hline & Especialização & 9 & 18 & 2 & 4 & 11 & 11 \\
\hline & Total & 50 & 100 & 50 & 100 & 100 & 100 \\
\hline \multirow[t]{6}{*}{ Estado civil } & Solteiro & 13 & 26 & 16 & 32 & 29 & 29 \\
\hline & Casado/Un. est. & 33 & 66 & 25 & 50 & 58 & 58 \\
\hline & Separado/divorciado & 2 & 4 & 4 & 8 & 6 & 6 \\
\hline & Viúvo & 2 & 4 & 2 & 4 & 4 & 4 \\
\hline & Outro & 0 & 0 & 3 & 6 & 3 & 3 \\
\hline & Total & 50 & 100 & 50 & 100 & 100 & 100 \\
\hline \multirow[t]{6}{*}{ Religião } & Cristã & 45 & 90 & 45 & 90 & 90 & 90 \\
\hline & Espiritualista & 0 & 0 & 3 & 6 & 3 & 3 \\
\hline & Nenhuma específica & 1 & 2 & 1 & 2 & 2 & 2 \\
\hline & Ateu-agnóstico-nenhuma & 2 & 4 & 0 & 0 & 2 & 2 \\
\hline & Não respondeu & 2 & 4 & 1 & 2 & 3 & 3 \\
\hline & Total & 50 & 100 & 50 & 100 & 100 & 100 \\
\hline \multirow[t]{3}{*}{ Trabalha } & Sim & 35 & 70 & 45 & 90 & 80 & 80 \\
\hline & Não & 15 & 30 & 5 & 10 & 20 & 20 \\
\hline & Total & 50 & 100 & 50 & 100 & 100 & 100 \\
\hline \multirow[t]{22}{*}{ Profissão } & Não respondeu & 1 & 2 & 4 & 8 & 5 & 5 \\
\hline & Administrador & 5 & 10 & 1 & 2 & 6 & 6 \\
\hline & Serviços gerais & 5 & 10 & 1 & 2 & 6 & 6 \\
\hline & Auxiliar administrativo & 1 & 2 & 2 & 4 & 3 & 3 \\
\hline & Vendas/relacionamento & 4 & 8 & 4 & 8 & 8 & 8 \\
\hline & Funcionário público & 1 & 2 & 1 & 2 & 2 & 2 \\
\hline & Estudante & 1 & 2 & 1 & 2 & 2 & 2 \\
\hline & Aposentado & 8 & 16 & 0 & 0 & 8 & 8 \\
\hline & Do lar & 4 & 8 & 2 & 4 & 6 & 6 \\
\hline & Serviços de informática & 1 & 2 & 1 & 2 & 2 & 2 \\
\hline & Artes e similares & 1 & 2 & 0 & 0 & 1 & 1 \\
\hline & Educação & 6 & 12 & 0 & 0 & 6 & 6 \\
\hline & Serviços de saúde & 4 & 8 & 27 & 54 & 31 & 31 \\
\hline & Mecânica/equipamentos & 0 & 0 & 1 & 2 & 1 & 1 \\
\hline & Serviços de segurança & 0 & 0 & 1 & 2 & 1 & 1 \\
\hline & Setor da alimentação & 1 & 2 & 0 & 0 & 1 & 1 \\
\hline & Engenharia & 1 & 2 & 1 & 2 & 2 & 2 \\
\hline & Agricultura & 3 & 6 & 2 & 4 & 5 & 5 \\
\hline & Autônomo & 2 & 4 & 0 & 0 & 2 & 2 \\
\hline & Transportes & 1 & 2 & 0 & 0 & 1 & 1 \\
\hline & Estética & 0 & 0 & 1 & 2 & 1 & 1 \\
\hline & Total & 50 & 100 & 50 & 100 & 100 & 100 \\
\hline \multirow[t]{7}{*}{ Renda familiar } & Entre 0 e $2 \mathrm{SM}$ & 13 & 26 & 11 & 22 & 24 & 24 \\
\hline & Entre 3 e $4 \mathrm{SM}$ & 5 & 10 & 21 & 42 & 26 & 26 \\
\hline & Entre 5 e $6 \mathrm{SM}$ & 17 & 34 & 13 & 26 & 30 & 30 \\
\hline & Entre 7 e $10 \mathrm{SM}$ & 6 & 12 & 5 & 10 & 11 & 11 \\
\hline & Entre 11 e $15 \mathrm{SM}$ & 4 & 8 & 0 & 0 & 4 & 4 \\
\hline & Não respondeu & 5 & 10 & 0 & 0 & 5 & 5 \\
\hline & Total & 50 & 100 & 50 & 100 & 100 & 100 \\
\hline
\end{tabular}

Fonte: primária. 
TABELA 2

Comparação do desempenho dos participantes nos instrumentos em função do município.

\begin{tabular}{|c|c|c|c|c|c|c|c|c|c|c|c|c|c|c|c|}
\hline \multirow[b]{2}{*}{ Teste } & \multirow[b]{2}{*}{ Dimensão } & \multicolumn{4}{|c|}{ Total } & \multicolumn{4}{|c|}{ Itá } & \multicolumn{4}{|c|}{ Passo Fundo } & \multicolumn{2}{|c|}{ Mann-Whitney } \\
\hline & & Mediana & Média & $D P$ & $\begin{array}{l}N C M \\
(95 \%)\end{array}$ & Mediana & Média & $D P$ & $\begin{array}{l}N C M \\
(95 \%)\end{array}$ & Mediana & Média & $D P$ & $\begin{array}{l}N C M \\
(95 \%)\end{array}$ & $U$ & $\begin{array}{c}p \\
\text { bicaudal }\end{array}$ \\
\hline CES-D & Total & 11,50 & 13,22 & 9,76 & 1,94 & 10,00 & 11,26 & 7,97 & 2,27 & 12,50 & 15,18 & 11,00 & 3,13 & 2290,50 & 0,11 \\
\hline \multirow[t]{8}{*}{ LIS-D } & Afetivos & 5,00 & 5,49 & 4,59 & 0,91 & 4,00 & 4,82 & 4,38 & 1,25 & 6,00 & 6,16 & 4,73 & 1,34 & 2317,50 & 0,15 \\
\hline & Comportamento & 5,00 & 5,95 & 4,57 & 0,91 & 5,00 & 5,34 & 4,61 & 1,31 & 6,50 & 6,56 & 4,49 & 1,28 & 2328,50 & 0,18 \\
\hline & Pensamento & 3,00 & 3,76 & 3,16 & 0,63 & 3,00 & 3,68 & 3,13 & 0,89 & 3,00 & 3,84 & 3,21 & 0,91 & 2494,50 & 0,83 \\
\hline & Somáticos & 7,00 & 7,21 & 4,52 & 0,90 & 6,50 & 6,64 & 4,01 & 1,14 & 8,00 & 7,78 & 4,95 & 1,41 & 2354,50 & 0,24 \\
\hline & Visão de si & 4,00 & 4,66 & 3,82 & 0,76 & 3,00 & 4,00 & 3,86 & 1,10 & 5,00 & 5,32 & 3,70 & 1,05 & 2243,00 & $0,05^{*}$ \\
\hline & Visão do mundo & 4,00 & 4,45 & 3,64 & 0,72 & 4,00 & 4,32 & 3,40 & 0,97 & 4,00 & 4,58 & 3,90 & 1,11 & 2500,50 & 0,87 \\
\hline & Visão de futuro & 3,00 & 3,66 & 3,94 & 0,78 & 3,00 & 3,30 & 3,60 & 1,02 & 3,00 & 4,02 & 4,26 & 1,21 & 2435,50 & 0,54 \\
\hline & Total & 31,00 & 35,18 & 23,63 & 4,69 & 27,50 & 32,10 & 22,51 & 6,40 & 36,50 & 38,26 & 24,53 & 6,97 & 2339,00 & 0,20 \\
\hline IDATE & Total & 37,00 & 38,09 & 9,95 & 1,97 & 34,50 & 35,78 & 9,06 & 2,57 & 38,50 & 40,40 & 10,35 & 2,94 & 2175,00 & $0,02 *$ \\
\hline LIS-A & Total & 21,50 & 25,76 & 21,09 & 4,18 & 21,50 & 24,88 & 18,75 & 5,33 & 21,00 & 26,64 & 23,35 & 6,64 & 2539,50 & 0,92 \\
\hline EEP & Total & 22,00 & 22,46 & 8,40 & 1,67 & 20,00 & 20,44 & 8,66 & 2,46 & 24,00 & 24,48 & 7,70 & 2,19 & 2184,50 & $0,02 *$ \\
\hline LIS-E & Total & 17,50 & 21,92 & 17,87 & 3,55 & 18,50 & 21,34 & 15,02 & 4,27 & 15,00 & 22,50 & 20,46 & 5,81 & 2591,00 & 0,65 \\
\hline
\end{tabular}

$\mathrm{NCM}=$ nível de confiança da média. A presença de * indica valores de $\mathrm{p} \leq 0,05$. Fonte: primária.

\section{Discussão}

A análise dos dados identificou que os escores de maior prejuízo em sintomas depressivos, de ansiedade e de estresse foram encontrados na amostra do município de comparação, embora dentro da faixa de baixos sintomas compatível com a população não-clínica. Esses resultados podem ser devidos a uma possível melhor qualidade de vida (QV) para cidades de menor porte, maior estabilidade populacional e um sentido de identidade entre as pessoas que fortalece os laços afetivos entre os sujeitos e reforça a interação social (Sequeira \& Silva, 2002). O município de comparação (Passo Fundo) possuía cerca de 183.300 habitantes em comparação com Itá, que tinha 6.417 habitantes (IBGE, 2007). É possível que o tamanho populacional do município possa ser uma variável que esteja interferindo na saúde mental das pessoas.

Em relação aos sintomas depressivos, houve diferença estatisticamente significativa apenas na visão de si do LIS-D, apontando que a amostra do município de comparação apresentou este aspecto menos favorável que a de Itá. A visão de mundo pode ser compreendida pela maneira como a pessoa define suas experiências com o ambiente (Beck, Rush, Shaw, \& Emery, 1997). Desta forma, os dados indicam que o município de menor porte parece estar favorecendo uma visão de si mais favorável, o que pode ter um impacto positivo na qualidade de vida.
Os resultados apresentados sugerem que a amostra de Itá tenha um ritmo de vida menos estressante do que a amostra do município de comparação. Os sintomas de estresse são situacionais, ou seja, é causado por eventos mais breves no tempo do que os sintomas de ansiedade e sintomas depressivos, como uma resposta que o organismo produz para adaptar-se a pressões internas e externas. Contudo, este processo é limitado no tempo, e em muitos casos o organismo entra na fase de esgotamento caso a adaptação não seja alcançada (Lentine, Sonoda, \& Biazin, 2003). Pode-se considerar que municípios maiores gerem mais vulnerabilidade ao estresse devido a maiores pressões e um ritmo de vida mais acelerado, e consequentemente dificulte o processo funcional de adaptação.

Não foram identificados sintomas depressivos, tríade cognitiva, sintomas de estresse e de ansiedade como efeitos residuais na população de Itá após 20 anos da instalação da barragem. De acordo com documentos da Eletrosul (1992), houve o impacto e preocupações iniciais com a notícia da inundação da cidade, porém não teria ocorrido uma reação negativa significativa contra esse processo. A população demonstrou aceitação com a nova cidade, que fora planejada de acordo com a arquitetura original (Viana, 2003), o que pode ter sido um importante fator protetivo para a população. A construção de barragens ocasiona impactos socioambientais, mas uma boa condução do processo de negociação e reparação para minimizar esses impactos para a população é essencial. No caso 
de Itá, destaca-se que a empresa desenvolveu vários planos compensatórios para diminuir os prejuízos, e foram realizados 23 programas socioambientais que incluíram trabalhos em vários setores, posto que o objetivo que direcionou esses projetos foi o de potencializar os efeitos positivos da construção e diminuir os impactos (Celestino, Phillips, \& Rocha, 2008). Cabe mencionar que a Igreja Matriz da cidade ficou com as torres para fora da água, numa lembrança da cidade que ficou sob o lago formado pela Usina Itá como um marco simbólico da antiga localização da cidade (Carvalho, 2006). Esses elementos podem ter auxiliado no processo de proteção do surgimento de sintomas psicológicos.

\section{Conclusões}

A prevalência de sintomas depressivos, tríade cognitiva, sintomas de ansiedade e estresse de uma população de um município que passou há 20 anos pelo processo de realocação para a instalação de uma barragem para UHE, em comparação com um município que não passou por este processo, foi considerada praticamente sem diferenças, inclusive sugerindo ser de melhor qualidade no caso estudado do que no município de comparação. É possível que estes achados sejam derivados de um melhor padrão de qualidade de vida de municípios de menor porte, favorecendo escores mais baixo de sintomas depressivos, de ansiedade e de estresse. Contudo, a diferença de tamanho dos municípios pode ter sido uma variável que influenciou na análise; a realização de estudos que comparem municípios de porte semelhante que passaram e que não passaram pelo processo de instalação de barragens e realocação permitirá uma identificação mais precisa da diferença de sintomas depressivos, de ansiedade e de estresse.

Não foi possível comparar os escores dos sintomas depressivo, tríade cognitiva, sintomas de ansiedade e de estresse atuais com o período imediatamente pós-realocação, visto que não são conhecidos estudos conduzidos naquela população. Desta forma, não foi possível determinar se estes escores estão relacionados com os fatores situacionais atuais (diferença do tamanho das cidades), se foi devido ao tempo ou a forma como houve a condução da transposição do município. É interessante considerar a inclusão de pesquisas sobre estes sintomas em populações pré e pós instalação das barragens, bem como acompanhamentos longitudinais, no sentido de poder se identificar com melhor precisão as alterações de sintomas depressivos, de ansiedade e de estresse eventualmente associados ao processo de instalação de barragens, pois permitirão aferir de forma mais precisa os impactos humanos e os investimentos necessários em saúde mental, para além dos investimentos sobre o impacto ambiental.

Um aprimoramento do processo de avaliação humano dos impactos ambientais da instalação de barragens faz-se necessária, e deve-se considerar a inclusão da avaliação de sintomas depressivos, tríade cognitiva, sintomas de ansiedade e de estresse como uma importante estratégia. O papel da psicologia nesta avaliação é fundamental, visto que estes sintomas são a porta de entrada para a instalação de transtornos mentais e de comportamento, o que implica no aumento de investimentos públicos na área da saúde. O que puder ser feito para se evitar isso contribuirá, em muito, para a economia com os investimentos em saúde e na promoção da qualidade de vida.

\section{Referências}

American Psychiatric Association. (2014). Manual diagnóstico e estatístico de transtornos mentais (DSM-5). Porto Alegre: Artmed.

Beck, A. T., Rush, A. J., Shaw, B. F., \& Emery, G. (1997). Terapia cognitiva da depressão. Porto Alegre: Artmed.

Borges, R. S. \& Silva, V. P. (2011). Usinas hidrelétricas no Brasil: a relação de afetividades dos atingidos com os lugares inundados pelos reservatórios. Caminhos da geografia, 12(40), 222-231.

Bronfenbrenner, Urie. (2002). A Ecologia do Desenvolvimento Humano: experimentos naturais e planejados. Porto Alegre: Artmed.

Carvalho, O. A. D. (2006). Água sobre terra: lugar e territorialidade na implantação de grandes hidrelétricas (Dissertação de mestrado). Recuperado de <http://www.lume.ufrgs.br/handle/10183/12041>.

Celestino, V. S. \& Phillips, J. W. \& Rocha, R. S. (2008). O Reordenamento Territorial em Grandes Obras: O Caso da Cidade de Itá. II Simpósio Brasileiro de Ciências Geodésicas e Tecnologias da Geoinformação. Recuperado de $<$ https://www.ufpe.br/cgtg/SIMGEOII_CD/Organizado/cad/212.pdf>.

Costa, G. B., Locks, R., \& Matos, D.. (2010). Análise do Relatório do Impacto Ambiental das Usinas Hidrelétricas no Rio Madeira no Município de Porto Velho/RO. V Encontro Nacional da Anppas. Recuperado de <http://www.anppas. org.br/encontro5/cd/artigos/GT14-344-287-20100902124004.pdfs. 
Dancey, C. P. \& Reidy, J. (2013). Estatística sem matemática para psicologia. Porto Alegre: Penso.

Elementos Culturais da Área do Reservatório da Usina Hidrelétrica Itá - Ecau. (2001). O Trabalho e os Dias - histórias de vida de antigos moradores da área da barragem da UHE - Itá. Programa Arca de Noé - Eletrosul, 10-60.

Fioravanti, A. C. M., Santos, L. D. F., Maissonette, S., Cruz, A. P. D. M., \& Landeira-Fernandez, J. (2006). Avaliação da estrutura fatorial da Escala de Ansiedade-Traço do IDATE. Avaliação Psicológica, 5(2), 217-224.

Ferreira, V. R. T. (2012). Levantamento da Intensidade de Sintomas Depressivos - LIS-D. Passo Fundo: não publicado.

Ferreira, V. R. T. (2015). Levantamento da Intensidade de Sintomas de Ansiedade - LIS-A. Passo Fundo: não publicado

Ferreira, V. R. T. (2015). Levantamento da Intensidade de Sintomas de Estresse - LIS-E. Passo Fundo: não publicado.

Fleck, M. P., Lousada, S., Xavier, M., Chachamovich, E., Vieira, G., Santos, L. \& Pinzon, V. (2000). Aplicação da versão em português do instrumento abreviado de avaliação da qualidade de vida WHOQOL-bref. Revista de Saúde Pública, 34(2), 178-183. http://dx.doi.org/10.1590/s0034-89102000000200012

Hall, F. \& Mattos, T. (1984). Memória Itá. Itá, s/e.

Hauck, N. F. \& Teixeira, M. A. P. (2011). A estrutura fatorial da Escala CES-D em estudantes universitários brasileiros. Avaliação Psicológica, 10(1), 91-97.

Hermes, J. (2013). Aspectos Históricos e Culturais do Município de Itá/SC - Capacitação de Monitores Casa da Cultura. Secretaria de Desenvolvimento Econômico e Departamento de Cultura. Itá, SC.

IBGE (2007). Instituto Brasileiro de Geografia e Estatística: Contagem da população (2007). Disponível em: <http:// www.ibge.gov.br/home/estatistica/populacao/contagem2007/populacao_2007_DOU_05_10_2007.xls>.

Kedia, S. \& Van Willigen, J. (2001). Effects of forced displacement on the mental health of older people in north India. Haley International Journal of Aging, 3(1), 81-83. <http://dx.doi.org/10.2190/CAAC-X21P-112R-Q5P3>.

Lancetti, A. \& Amarante, P. (2006). Saúde mental e saúde coletiva. In Campos, G. W. D. S., Minayo, M. D. S., Akerman, M., Drumond Júnior, M., \& Carvalho, Y. D. Tratado de saúde coletiva (pp. 615-634). São Paulo: Hucitec.

Lentine, E. C., Sonoda, T. K., \& Biazin, D. T. (2003). Estresse de Profissionais de Saúde das Unidades Básicas do Município De Londrina. Terra e Cultura, 37, 103-123.

Levin, J. \& Fox, J. A. (2004). Estatística para ciências humanas. São Paulo: Pearson.

Lopes, P. A. (1999). Probabilidade e estatística. Rio de Janeiro: Reichmann \& Affonso.

Luft, C., Sanches, S., Mazo, G., \& Andrade, A. (2007). Versão brasileira da escala de estresse percebido: tradução e validação para idosos. Rev. Saúde Pública, 41(4), 606-15. <http://dx.doi.org/10.1590/S0034-89102007000400015>.

Magalhães, S. C. G. (2006). Estudo dos Impactos sociais e ambientais decorrentes dos projetos hidrelétricos de Jirau e Santo Antônio - Reflexões preliminares. Mestrado em Desenvolvimento Regional e Meio Ambiente. Rondônia: Unir. PGDRA, 1-15.

Mendonça, L. (2011). O legado das hidrelétricas. O Setor Elétrico (São Paulo), (68), 1.

Passos, R. M. \& Praxedes, N. (2013). Reinserção em Nova Mutum Paraná e Condições de Vida dos Atingidos pela Construção da Hidrelétrica de Jirau em Rondônia. Revista de Administração e Negócios da Amazônia, 5(1), 1-12.

Pinto, L. C. (2012). Os projetos hidrelétricos como causa dos deslocamentos populacionais: migrações forçadas em nome do desenvolvimento. Lisboa: Faculdade de Ciências Sociais e Humanas, Universidade Nova de Lisboa. (pp. 1-123).

Pirta, R., Chandel, N., \& Pirta, C. (2014). Attachment and Displacement: The Resettlers of Bhakra Dam are Hurt. Psychological Studies, 59(1), 1-10. http://dx.doi.org/10.1007/s12646-013-0211-0

Relocação da Sede Municipal de Itá. (1984). Plano de Mudança. Itá.

Rodrigues, A. L. \& França, A. C. L. (2010). Uma perspectiva psicossocial em psicossomática via estresse e trabalho. In Mello Filho, J. (Org.). Psicossomática Hoje (Cap. 10, pp. 112-134).

Rossouw, N. (2008). Verifying the social impacts of the Berg river dam: An ex-post analysis. International Association of Impact Assessment. Recuperado de: <https://www.researchgate.net/profile/Nigel_Rossouw/publication/228759999 VERIFYING_THE_SOCIAL_IMPACTS_OF_THE_BERG_RIVER_DAM_AN_EX-POST_ANALYSIS/ links/5565928 $\overline{\mathrm{e} 0} 08 \mathrm{aec} \overline{4} \mathrm{~b} 0 \mathrm{f} 485 \mathrm{a} 2 \overline{\mathrm{b}} 6 . \mathrm{pdf}$.

Sadock, B. J., Sadock, V. A., \& Ruiz, P. (2017). Compêndio de Psiquiatria: Ciência do Comportamento e Psiquiatria Clínica. Porto Alegre: Artmed.

Sequeira, A. \& Silva, M. N. (2002). O bem estar da pessoa idosa no meio rural. Análise Psicológica, 20(3), 505-516.

Skinner, B. F. (2003). Ciência e comportamento humano. São Paulo: Martins Fontes.

Silveira, D. X. da \& Jorge, M. R. (1998). Propriedades psicométricas da escala de rastreamento populacional para depressão CES-D em populações clínica e não-clínica de adolescentes e adultos jovens. Revista de Psiquiatria Clínica, 25(5), 251-261.

Souza, W. L. (2000). Impacto Ambiental de Hidrelétricas: Uma Análise Comparativa de Duas Abordagens. Rio de Janeiro: UFRJ.

Tractebel Energia - GDF Suez. (2015). UHE Itá. Itá. 
Vainer, C. \& Vieira, F. B. (2005). Movimento dos Atingidos por Barragens. Manual do atingido: Uma história de organização e luta. Laboratório Estado, Trabalho, Território e Natureza/ Instituto de Pesquisa e Planejamento Urbano e Regional. Rio de Janeiro: UFRJ.

Viana, R. M. (2003). Grandes Barragens, Impactos e Reparações: Um Estudo De Caso Sobre A Barragem De Itá. Instituto de Pesquisa e Planejamento Urbano e Regional. Rio de Janeiro: UFRJ.

Dados dos autores:

Franciele Luísa Pozzebon - Especialista, Faculdade Meridional.

Vinícius Renato Thomé Ferreira - Doutor, coordenador do Programa de Mestrado em Psicologia da IMED.

Endereço para correspondência:

Vinícius Renato Thomé Ferreira

Av. Sete de Setembro, 759, bloco A apto 701

99010-121, Passo Fundo, RS, Brasil

<vthome2@gmail.com>

Recebido em: 28.03.2017

Aceito em: 14.09.2017 